\title{
Optical imaging of post-embryonic zebrafish using multi orientation raster scan optoacoustic mesoscopy
}

\author{
Murad Omar ${ }^{1,2, *}$, Johannes Rebling ${ }^{1,2, *}$, Kai Wicker ${ }^{3, *}$, Tobias Schmitt-Manderbach ${ }^{3}$, Mathias Schwarz ${ }^{1,2}$, \\ Jérôme Gateau ${ }^{4}$, Hérnan López-Schier ${ }^{5}$, Timo Mappes $^{6}$ and Vasilis Ntziachristos ${ }^{1,2}$
}

\begin{abstract}
Whole-body optical imaging of post-embryonic stage model organisms is a challenging and long sought-after goal. It requires a combination of high-resolution performance and high-penetration depth. Optoacoustic (photoacoustic) mesoscopy holds great promise, as it penetrates deeper than optical and optoacoustic microscopy while providing high-spatial resolution. However, optoacoustic mesoscopic techniques only offer partial visibility of oriented structures, such as blood vessels, due to a limited angular detection aperture or the use of ultrasound frequencies that yield insufficient resolution. We introduce $360^{\circ}$ multi orientation (multi-projection) raster scan optoacoustic mesoscopy (MORSOM) based on detecting an ultra-wide frequency bandwidth (up to $160 \mathrm{MHz}$ ) and weighted deconvolution to synthetically enlarge the angular aperture. We report unprecedented isotropic inplane resolution at the 9-17 $\mu \mathrm{m}$ range and improved signal to noise ratio in phantoms and opaque 21-day-old Zebrafish. We find that MORSOM performance defines a new operational specification for optoacoustic mesoscopy of adult organisms, with possible applications in the developmental biology of adulthood and aging.
\end{abstract}

Light: Science \& Applications (2017) 6, e16186; doi:10.1038/Isa.2016.186; published online 13 January 2017

Keywords: deconvolution; development; mesoscopy; multiview; optoacoustics; photoacoustics

\section{INTRODUCTION}

Model organisms, such as Zebrafish and Drosophila melanogaster, are widely used in developmental biology and experimental genetics ${ }^{1,2}$. Although they are important biological models, optical imaging in these samples is often limited to the embryonic stage due to optical diffusion $^{3}$. In this embryonic stage, the samples are typically small in size and virtually transparent and they can be imaged in vivo using single-plane illumination microscopy (SPIM) ${ }^{3}$. Optical microscopy of larger specimens requires optical clearing using chemicals that are toxic and not suited for in vivo imaging. Alternative methods that enable intra-vital microscopy include confocal, multi-photon ${ }^{4,5}$ or optoacoustic microscopy ${ }^{6}$ techniques, which are nevertheless limited to depths of the order of a few hundred microns due to the physical limitations of focusing a light beam deep within highly scattering media ${ }^{4}$.

The imaging of larger opaque biological specimens is important for longitudinal observations of biological processes in vivo ${ }^{1,2,7}$. Imaging beyond the embryonic stage can allow observations of processes associated with adult development and aging. Raster scan optoacoustic mesoscopy $(\mathrm{RSOM})^{8,9}$ has been recently introduced for imaging model organisms and disease development, such as tumor growth in mice $^{10}$. The method has been used to image samples at depths of up to
$5 \mathrm{~mm}$ with a resolution of $4-20 \mu \mathrm{m}^{8,9}$, bridging the imaging abilities of microscopic and macroscopic imaging techniques, such as multispectral optoacoustic tomography ${ }^{11-13}$. However, RSOM of model organisms, such as Zebrafish, is limited due to the low numerical aperture (NA) of the ultrasonic detectors used, typically $\sim 60^{\circ}$. Consequently, RSOM exhibits highly anisotropic resolution in the $x y$-plane, which only partially detects directional objects ${ }^{8,14}$ and may miss certain structures (Supplementary Fig. S1).

Here, we introduce multi orientation RSOM (MORSOM), a technique that collects RSOM projections over $360^{\circ}$ angles, analogous to X-ray computed tomography (CT). In contrast to multiview optoacoustic microscopy ${ }^{6}$, which utilizes focused light beams and image formation based on optical diffraction, MORSOM utilizes broad-field illumination and forms images based on the ultrasonic diffraction limit. For this reason, a MORSOM-specific feature is the use of ultra-wideband detection (up to $160 \mathrm{MHz}$ ) to achieve high resolution and a broad coverage of spatial frequencies, which are necessary to capture high-quality images. This feature contrasts optoacoustic mesoscopy studies performed with narrowband, high-element-pitch linear array detectors ${ }^{15-17}$. Finally, image reconstruction schemes are necessary to produce the optimal combination of the MORSOM projections collected.

${ }^{1}$ Chair for Biological Imaging, Technische Universität München, München D-81675, Germany; ${ }^{2}$ Institute for Biological and Medical Imaging, Helmholtz Zentrum München, Neuherberg D-85764, Germany; ${ }^{3}$ Carl Zeiss AG, Corporate Research and Technology, Jena D-07745, Germany; ${ }^{4}$ ESPCI Paris, PSL Research University, CNRS, INSERM, Institut Langevin, Paris F-75005, France; ${ }^{5}$ Research Unit Sensory Biology and Organogenesis, Helmholtz Zentrum München, Neuherberg D-85764, Germany and ${ }^{6}$ Carl Zeiss Vision International GmbH, Technology \& Innovation, Aalen D-73430, Germany

*These authors contributed equally to this work.

Correspondence: M Omar, Email: murad.omar@tum.de; V Ntziachristos, Email: v.ntziachristos@tum.de

Received 20 February 2016; revised 1 July 2016; accepted 6 July 2016; accepted article preview online 11 July 2016 
We hypothesized that MORSOM could yield unprecedented imaging performance over RSOM while allowing penetration depths that are superior to optical resolution microscopy ${ }^{6}$. We developed a MORSOM experimental setup and investigated the performance with phantoms and 21 days post-fertilization (dpf) Zebrafish, a target tissue that has not been possible to image with $\mathrm{SPIM}^{18}$ or optical resolution optoacoustic microscopy ${ }^{6,10,19}$, to investigate this hypothesis. We further investigated the relative performance of MORSOM compared with conventional RSOM.

\section{MATERIALS AND METHODS}

\section{System design}

We present the experimental setup in Figure 1. The setup was designed to accommodate imaging of model organisms. The sample is positioned along the axis of rotation, the $z$ axis, by placing it on a rotation stage. The spherically focused detector was scanned in the $x z$ plane using two linear stages (M683 and M404-2PD, Physik Instrumente $\mathrm{GmbH}$, Karlsruhe, Germany). We scanned the sample in an alternating continuous-discrete manner, where the $\mathrm{x}$-stage is continuously moved and the z-stage is moved stepwise from one line to the next to acquire each orientation. At the end of every $x z$-scan, the sample was rotated $360^{\circ}$ using a rotational stage (RS-40, PI-MICOS, Karlsruhe, Germany).

Optical excitation was based on 532-nm, a 1-ns pulse width and 2-kHz repetition rate laser (Bright Solutions SRL, Pavia, Italy) emitting $1 \mathrm{~mJ}$ pulse $^{-1}$. Light was directed to the specimen via fibers (Ceram Optec GmbH, Bonn, Germany), achieving coupling efficiency of $\sim 30 \%$. The light intensity delivered to the sample was $\sim 0.32 \mathrm{~mJ} \mathrm{~cm}^{-2}$, which is approximately two orders of magnitude less than the ANSI limit for biological samples. We placed four fiber arms $90^{\circ}$ apart at a distance of $14.5 \mathrm{~mm}$ from the axis of rotation to generate homogeneous illumination of the sample (Supplementary Fig. S2).
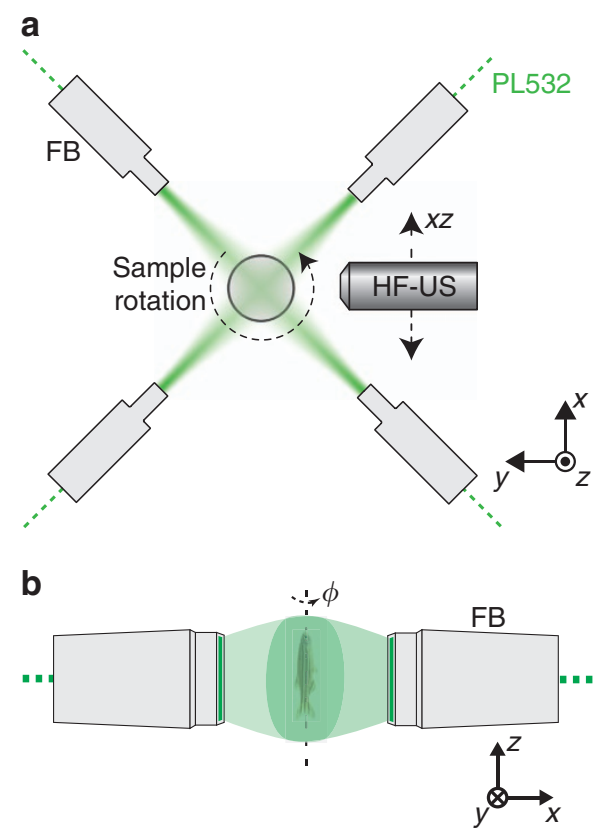

Figure 1 (a) Top view of the system showing the configuration of the fiber bundles, the location of the ultrasound detector, the scanning the $x z$ plane and the rotation around the $z$ axis. (b) Side view of the system showing the sample orientation and illumination.
Detection was based on custom-made single-element detectors with ultra-wideband detection. A detector with a nominal central frequency of $50 \mathrm{MHz}$ attained a focal distance of $3 \mathrm{~mm}$, an NA of $\sim 0.5$ and ultra-wide band acoustical $-6-\mathrm{dB}$ bandwidth of $23-115 \mathrm{MHz}$ (Supplementary Fig. S3a). A second detector with a nominal central frequency of $100 \mathrm{MHz}$ attained acoustical $-6-\mathrm{dB}$ bandwidth of $20-180 \mathrm{MHz}$, a focal distance of $1.65 \mathrm{~mm}$ and an NA of $\sim 0.45$. The detectors were connected to a $63-\mathrm{dB}$ low-noise amplifier (AU-1291, Miteq Inc., Hauppauge, NY, USA). The signals were digitized using a fast data acquisition card (ADQ412-3G, SP-Devices, Linköping, Sweden) at $900 \mathrm{MS} \mathrm{s}^{-1}$ and 12 bit resolution. The minimum angular step size $\left(\Delta \phi_{\min }\right)$ required to ensure that all the optoacoustic waves generated within the sample and in the xy plane are captured is given by the angle of acceptance of the detector. Consequently, we chose a rotation step of $\Delta \phi=20^{\circ}$ to enhance the signal to noise ratio (SNR) and to implement the same number of rotation steps when comparing the $50-\mathrm{MHz}$ and the $100-\mathrm{MHz}$ data (Supplementary Fig. S1). This choice leads to $N_{\phi}=18$ orientations. Detector scanning was performed along the $x z$ plane with a $20-\mu \mathrm{m}$ step size for the $50-\mathrm{MHz}$ case and a $10-\mu \mathrm{m}$ step size for the $100 \mathrm{MHz}$ case.

\section{Samples and preparation}

We characterized the MORSOM imaging performance in terms of resolution, SNR, and overall image quality using a phantom consisting of $10-\mu \mathrm{m}$ spheres that were randomly dispersed in agar gel and juvenile Zebrafish at $21 \mathrm{dpf}$. At $21 \mathrm{dpf}$, Zebrafish have a length of $\sim 10 \mathrm{~mm}$ and diameters ranging from 1 to $2 \mathrm{~mm}$ (Supplementary Fig. S4). These dimensions are not accessible by optical or optoacoustic microscopy techniques, such as SPIM $^{18}$ or multiview optical resolution photoacoustic microscopy $y^{6,19,20}$, and correspond to dimensions for which optoacoustic mesoscopy may be ideally suited. Zebrafish were embedded in low melting agar (Sigma-Aldrich, St Louis, MO, USA) and imaged ex vivo, consistent with the current regulations and processes allowed by the Government of Upper Bavaria for adult Zebrafish. The agar was mixed with $\sim 10 \mu \mathrm{m}$ diameter black microspheres (black polystyrene microspheres, Polybead, Polysciences Inc., Warrington, PA, USA), which we use as fiducial markers to confirm the exact alignment of the different RSOM projections onto the common MORSOM image reconstruction scheme. The Zebrafish/agar specimen was mounted on a syringe, as previously described for SPIM $^{21}$, and placed in a water bath for acoustic coupling.

\section{Reconstruction and deconvolution}

Planar raster scans were reconstructed separately in the local coordinates of the scan using three-dimensional beamforming with a dynamic aperture ${ }^{8,9}$. For accelerated performance, we parallelized the reconstruction on a graphical processing unit. The image grids were composed of $20 \times 5 \times 20 \mu \mathrm{m}^{3}$ voxels.

\section{Alignment of the individual projections}

After reconstruction in the local coordinates $\left(x^{\prime}, y^{\prime}, z^{\prime}\right)$, we transformed the three-dimensional reconstructions to the global coordinates $(x, y, z)$ with voxel sizes of $5 \times 5 \times 20 \mu \mathrm{m}^{3}$ through a linear transformation including two translations and a rotation. For example, the image $I_{m}\left(x^{\prime}, y^{\prime}, z^{\prime}\right)$ from the $m$ th orientation was transformed to the global coordinates as: $I_{m}(x, y, z)=T\left\{I_{m}\left(x^{\prime}, y^{\prime}, z^{\prime}\right), \Delta x, \Delta y, \phi_{m}\right\}$, where $\phi_{m}$ is the angle of rotation and $(\Delta x, \Delta y)$ is a shift vector required to account for location of the rotation axis in the reconstructed volume. We could only estimate the vector coordinates from the mechanical positioning; thus, we performed a calibration 
procedure to determine the exact vector coordinates. This calibration procedure was based on the reconstruction of the previously embedded $10 \mu \mathrm{m}$ microspheres, our fiducial markers. We selected the vector coordinates using the criterion of maximum brightness, which corresponds to the most coherent sum from all the orientations. We performed a fine calibration using cross-correlations in the spatial Fourier domain to improve the co-registration of the individual orientations. This fine calibration is an important step, as it allows sub-pixel calculation of the vector coordinates and corrects for differences in the shift vectors between different orientations as well as for the tilt of the scanning plane.

\section{Weighted sum and Wiener filtering}

The simplest way to combine the images from the individual views $\left(I_{m}\left(x, y ; z_{i}\right)\right)$ into a final image $\left(I\left(x, y ; z_{i}\right)\right)$ would be a simple summation, $I\left(x, y ; z_{i}\right)=\sum_{m=1}^{N_{\phi}} I_{m}\left(x, y ; z_{i}\right)$, or a summation in spatial frequency space, $\tilde{I}\left(k_{x}, k_{y} ; z_{i}\right)=\sum_{m=1}^{N_{\phi}} \tilde{I}_{m}\left(k_{x}, k_{y} ; z_{i}\right)$. This procedure is shown for a bead in Figure 2d. However, this reconstruction is not ideal because it does not take into account the different optical transfer functions (OTF, the Fourier transform of the point spread function (PSF) or $\operatorname{OTF}_{m}\left(k_{x}, k_{y}\right)$ of the individual views (Figure 2b)):

$$
\tilde{I}_{m}\left(k_{x}, k_{y} ; z_{i}\right)=\tilde{S}\left(k_{x}, k_{y} ; z_{i}\right) \times \operatorname{OTF}_{m}\left(k_{x}, k_{y}\right)
$$

where $\tilde{S}\left(k_{x}, k_{y} ; z_{i}\right)$ is the two-dimensional frequency representation of the sample absorber distribution for the slice $z=z_{i}$. At any given spatial frequency, both the amplitude and phase can vary between the OTFs of the different views. This variation influences the result of the summation in two ways. First, for varying phases, the sum of all OTFs will have a lower value than it would have if all phases were equal, leading to a reduced SNR. Second, at spatial frequencies where the amplitude of a view's OTF is low, this view cannot contribute much information to the summary image, although it will still add its noise components, again leading to a reduced SNR.

Thus, we calculated a weighted sum in the Fourier space ${ }^{22}$ to reduce the noise propagated from one view to the neighboring ones during reconstruction:

$$
\begin{aligned}
\tilde{I}_{\mathrm{WS}}\left(k_{x}, k_{y} ; z_{i}\right) & =\sum_{m=1}^{N_{\phi}} \tilde{w}_{m}\left(k_{x}, k_{y}\right) \tilde{I}_{m}\left(k_{x}, k_{y} ; z_{i}\right) \\
& =\sum_{m=1}^{N_{\phi}} \tilde{w}_{m}\left(k_{x}, k_{y}\right) \tilde{S}\left(k_{x}, k_{y} ; z_{i}\right) \operatorname{OTF}_{m}\left(k_{x}, k_{y}\right)
\end{aligned}
$$

where $\tilde{w}_{m}\left(k_{x}, k_{y}\right)$ are the view-dependent weight maps. These weight maps are chosen as the complex conjugate of the view's OTFs: $\tilde{w}_{m}\left(k_{x}, k_{y}\right)=\operatorname{OTF}_{m}^{*}\left(k_{x}, k_{y}\right)$. This calculation has two effects. First, it compensates for any unwanted phase effects in the OTFs:

$$
\begin{aligned}
\tilde{I}_{\mathrm{WS}}\left(k_{x}, k_{y} ; z_{i}\right) & =\sum_{m=1}^{N_{\phi}} \operatorname{OTF}_{m}^{*}\left(k_{x}, k_{y}\right) \tilde{I}_{m}\left(k_{x}, k_{y} ; z_{i}\right) \\
& =\sum_{m=1}^{N_{\phi}} \tilde{S}_{m}\left(k_{x}, k_{y} ; z_{i}\right)\left|\operatorname{OTF}_{m}\left(k_{x}, k_{y}\right)\right|^{2}
\end{aligned}
$$

Second, it weights a view's contribution at a certain frequency according to the amplitude of its OTF. Therefore, views with low transfer strengths (i.e., poor SNR) will contribute very little, whereas views with strong transfer strengths (i.e., good SNR) will contribute strongly. By choosing the weight maps in this manner, the information contained in the different view images will be combined in an SNRoptimized way. It can also compensate for phase shifts due to the electric impulse response of the detector.

This recombined image corresponds to the sample slice being imaged with an effective OTF:

$$
\tilde{I}_{\mathrm{WS}}\left(k_{x}, k_{y} ; z_{i}\right)=\tilde{S}_{m}\left(k_{x}, k_{y} ; z_{i}\right) \mathrm{OTF}_{\mathrm{WS}}\left(k_{x}, k_{y}\right)
$$
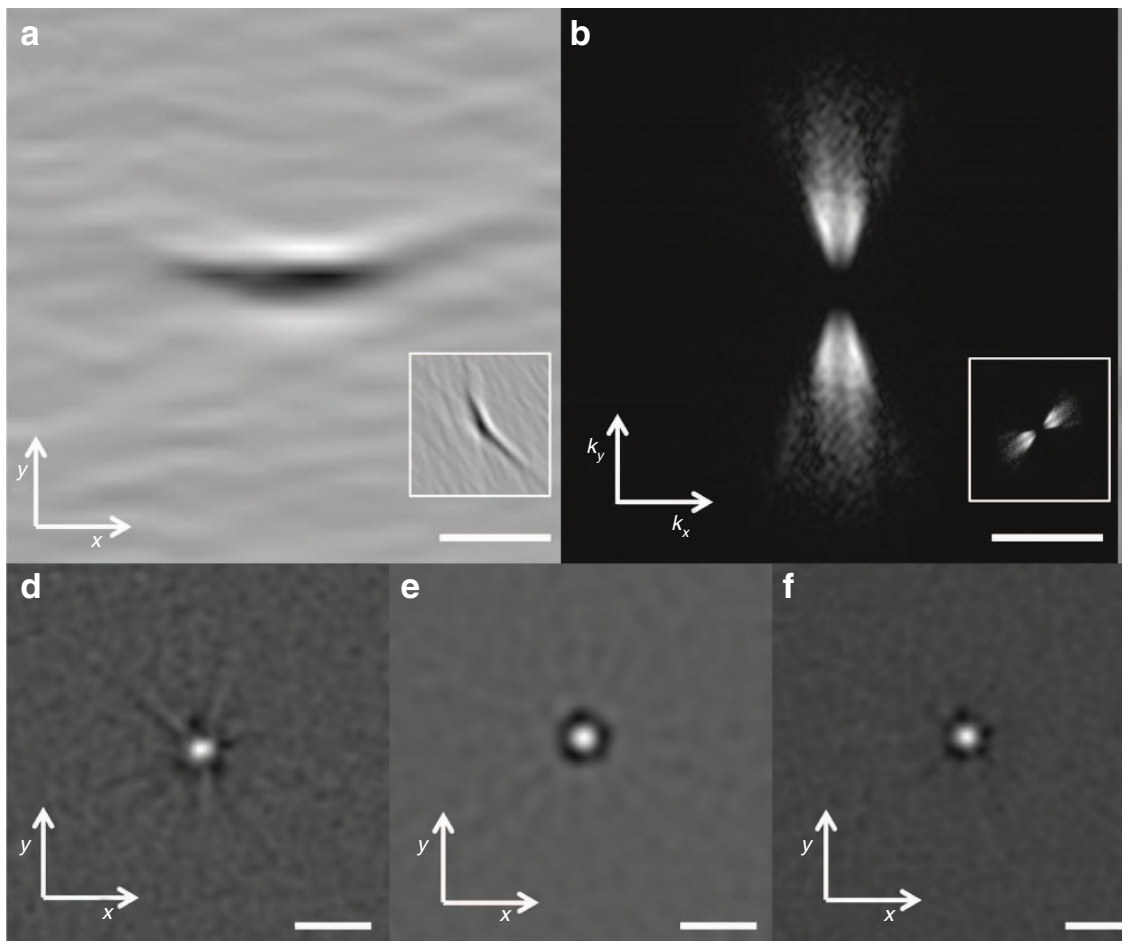
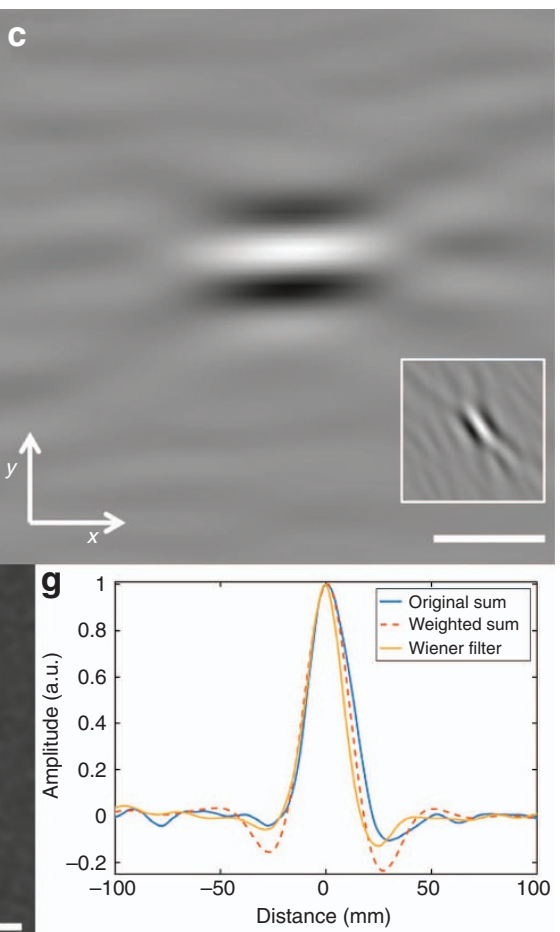

Figure 2 (a) PSF of an RSOM projection in the $x y$ plane at the $0^{\circ}$ angle; the inset shows the PSF at $60^{\circ}$. (b) Magnitude of the 0 TF of RSOM at $0^{\circ}$ and $60^{\circ}$, inset. (c) An image of a sphere corrected with the OTF in the frequency domain at $0^{\circ}$ and $60^{\circ}$, inset. (d) Multiview reconstruction of a microsphere. (e) Multiview reconstruction using a weighted sum. (f) Multiview reconstruction using a weighted sum and the Wiener filtering. (g) Cross-sections through the reconstructions in d-f. (Scale bars $=100 \mu \mathrm{m}$ ). 
with $\operatorname{OTF}_{\mathrm{WS}}\left(k_{x}, k_{y}\right)=\sum_{m=1}^{N_{\phi}}\left|\operatorname{OTF}_{m}\left(k_{x}, k_{y}\right)\right|^{2}$. The result of this process is shown for a bead in Figure 2e.

Finally, the resulting image can be further enhanced by applying a generalized Wiener filter ${ }^{22,23}$, which leads to a reduction of the side lobes (Figure 2f):

$$
\tilde{I}_{\text {Wiener }}\left(k_{x}, k_{y} ; z_{i}\right)=\frac{\tilde{I}_{\mathrm{WS}}\left(k_{x}, k_{y} ; z_{i}\right) \times \operatorname{OTF}_{\mathrm{WS}}^{*}\left(k_{x}, k_{y}\right)}{\left|\operatorname{OTF}_{\mathrm{WS}}\left(k_{x}, k_{y}\right)\right|^{2}+\omega^{2}}
$$

In the actual reconstructions, we empirically chose the Wiener factor $\omega$ as $10^{-3}$. The OTF was calculated as a two-dimensional Fourier transform of the PSF, which was experimentally determined, as described in the Supplementary Information.

\section{RESULTS AND DISCUSSION}

MORSOM images from a phantom of $10-\mu \mathrm{m}$ black microspheres suspended in agar obtained with 18 RSOM projections (Figure 2) were used to characterize the imaging performance. Figure $2 \mathrm{a}$ shows a single-projection image (RSOM) revealing the PSF for the $50-\mathrm{MHz}$ detector. Deconvolution of the RSOM images with the OTF (Figure 2b) results in noise suppression and phase correction (Figure 2c) compared with an original RSOM projection (Figure 2a). Figure $2 \mathrm{~d}$ shows a MORSOM image resulting from the direct summation of the different RSOM projections after crosscorrelation based sub-pixel co-registration, which enhances the SNR by $6 \mathrm{~dB}$. The use of a weighted sum of the different orientations (projections) suppresses the imaging artifacts (Figure 2e) in Figure 2d. Finally, the application of the Wiener filter (Figure 2f) leads to a further suppression of imaging artifacts compared with both Figure $2 \mathrm{~d}$ and $2 \mathrm{e}$, which is evidenced by the reduction of the negative values and streak artifacts. The use of the Wiener filter further enhances the SNR by $9 \mathrm{~dB}$ over non-weighted MORSOM reconstruction, for a total
15- $\mathrm{dB}$ improvement in SNR compared with the performance of the single-projection RSOM.

The theoretical calculations predicted ${ }^{24,25}$ (Supplementary Information) that the resolution of the $50-\mathrm{MHz}$ MORSOM system was $16 \mu \mathrm{m}$ in the $x y$ plane and $43 \mu \mathrm{m}$ along the axis of rotation (the $z$ axis). Cross-sections taken through one of the microspheres in Supplementary Fig. S3b after reconstruction revealed that the resolution along the $z$ axis $\left(\delta_{z}\right)$ was $44 \mu \mathrm{m}$ (full width at half maximum), and the resolution within the $x y$ plane $\left(\delta_{x y}\right)$ improved by a factor of $\sim 3$ along the $y$ axis over single-projection RSOM to $\sim 17 \mu \mathrm{m}$. The corresponding in-plane resolution for $100-\mathrm{MHz}$ MORSOM was $\sim 10 \mu \mathrm{m}$.

Figure 3 presents maximum intensity projection (MIP) images of MORSOM and RSOM from the $21 \mathrm{dpf}$ Zebrafish at $23-115 \mathrm{MHz}-$ bandwidth (50-MHz detector). As with the microsphere measurements, we noticed an improvement in the SNR of the MORSOM images compared with the RSOM images. Figure $3 \mathrm{a}$ shows a lateral image of the Zebrafish imaged with MORSOM and Figure $3 \mathrm{~b}$ shows the same lateral image acquired using RSOM. A comparison of the two images reveals enhancements in terms of contrast and SNR for MORSOM compared with RSOM. Marked differences are particularly evident at the dorsal side of the fish (Figure $3 \mathrm{c}$ and $3 \mathrm{~d}$ ), i.e., the fish sides that were not facing the detector. The differences between MORSOM and RSOM may be better understood by observing crosssections through the Zebrafish (axial images in Figure $3 \mathrm{e}$ and $3 \mathrm{f}$ ). MORSOM reveals anatomical features with unparalleled clarity compared with the single orientation images acquired with RSOM. Structures such as the boundary of the Zebrafish and the boundary of some internal organs are well visualized in MORSOM, but fail to be reconstructed by RSOM, the latter revealed almost none of the internal organs. We can pinpoint several examples of enhancement; for example, labels 1 and 3 point to the boundary of internal organs inside the Zebrafish. This boundary is clearly visible in Figure 3e, but it is incomplete in Figure 3f. Another example is labels 5 and 6; these

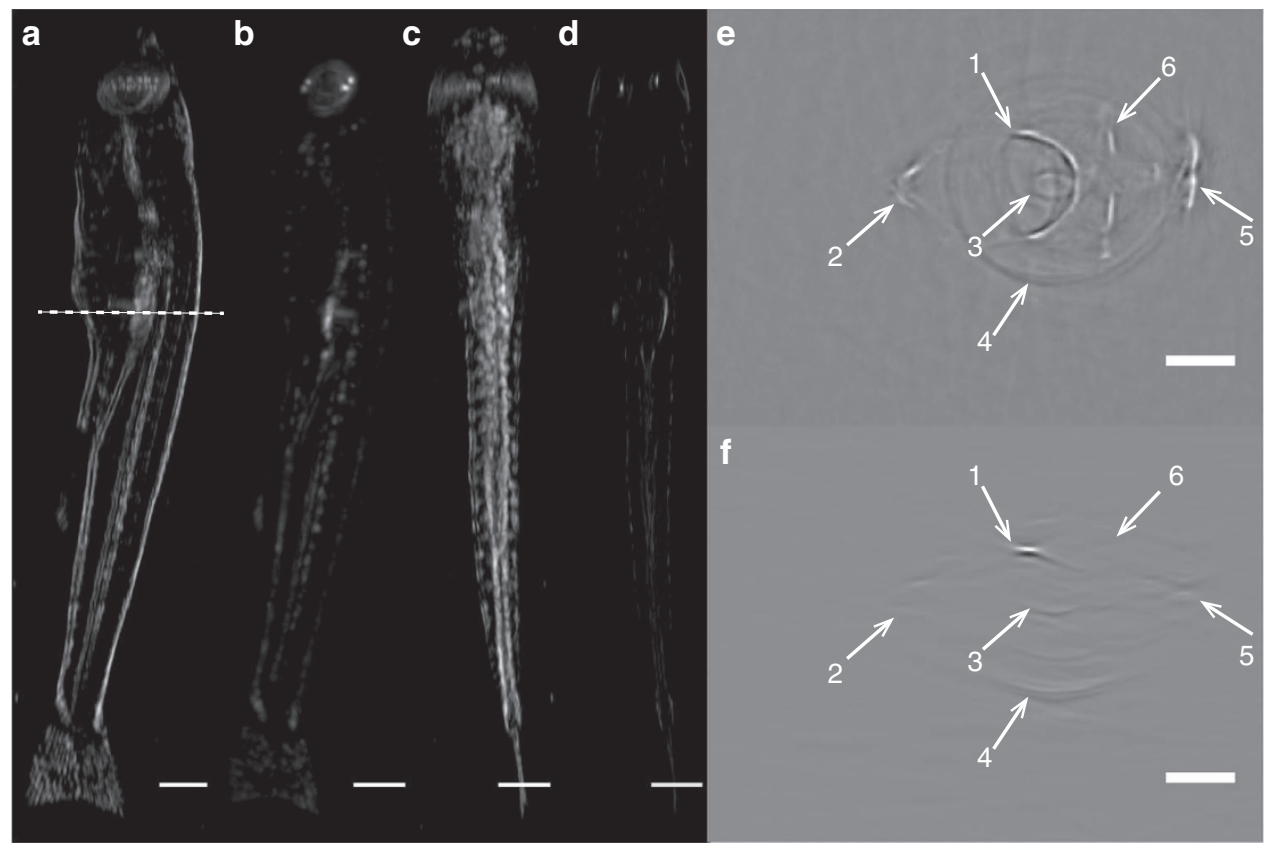

Figure 3 Comparison of the images generated using MORSOM and RSOM. (a) Top view MIP from MORSOM. (b) Top view MIP from RSOM. (c) Side view MIP from MORSOM. (d) Side view MIP from RSOM. (e) Cross section through the Zebrafish taken from MORSOM at the position indicated by the broken line in a. (f) Same cross section from RSOM. The arrows point to the similar features shown in e and $\mathbf{f}$. In the MORSOM case, many features are either complete or do not even appear in $\mathbf{f}$ (Scale bars $=\mathbf{a}-\mathbf{d} 500 \mu \mathrm{m}$; e,f $250 \mu \mathrm{m}, 0.3 \%$ of the pixels in a-d were saturated to improve the visibility of the RSOM images). 
two labels point to structures that are only visible in Figure 3e, i.e., in MORSOM. The cross-sections generated from MORSOM correspond well with the distribution of structures inside the Zebrafish ${ }^{26}$. For example, the eyes, pigmentation, fins, spinal cord and pharyngeal cartilage are clearly observed.

A comparison of MORSOM imaging at 50 and $100 \mathrm{MHz}$ (Figure 4) revealed enhanced resolution and better differentiation of anatomical features when using the higher frequency detector. Figure $4 \mathrm{a}$ and $4 \mathrm{~b}$ shows MIPs taken from the dorsal side of a Zebrafish using the 50 - and the $100-\mathrm{MHz}$ detectors, respectively. The lateral fish sides imaged by the 50 - and $100-\mathrm{MHz}$ detectors are shown in Figure $4 \mathrm{c}$ and $4 \mathrm{~d}$, respectively, and cross-sections taken with the two detectors are shown in Figure $4 \mathrm{e}$ and $4 \mathrm{f}$, respectively. The images confirm the enhanced resolution achieved with the $100-\mathrm{MHz}$ detector (acoustic bandwidth $20-180 \mathrm{MHz}$ ) compared with the $50-\mathrm{MHz}, 92-\mathrm{MHz}-$ bandwidth case. On the other hand, the $50-\mathrm{MHz}$ detector yields images with a higher signal to noise ratio and thus higher sensitivity to weak absorbers. Due to the increased SNR and coarser step size, 50$\mathrm{MHz}$ imaging required $20 \mathrm{~min}$ of acquisition time compared with $80 \mathrm{~min}$ for the $100-\mathrm{MHz}$ system. Therefore, the choice between the $50-\mathrm{MHz}$ detector and the $100-\mathrm{MHz}$ detector imposes a trade-off between sensitivity, scanning time and resolution. We expect that the 160-MHz-bandwidth detection would be better suited for imaging finer anatomical features, such as specimens where higher resolution is required. Conversely, the $92-\mathrm{MHz}$-bandwidth detector may be more appropriate for resolving spectral features with a higher SNR when multi-wavelength imaging is implemented.

Although single band images were shown here, multi-frequency band images could be generated for a higher fidelity and better representation of low- and high-spatial frequencies ${ }^{17}$. These images can be obtained by separately processing the frequency sub-bands from the ultra-wide ultrasound frequency spectrum collected.

\section{CONCLUSIONS}

We combined optoacoustic imaging over $360^{\circ}$ projections with ultrawide band detection ranging from 92 to $160 \mathrm{MHz}$ to achieve high performing optoacoustic mesoscopy. The method synthetically captures the optoacoustic waves propagating at multiple projections normal to the axis of rotation. The use of multiview approaches is common in many imaging modalities, including optical resolution optoacoustic microscopy ${ }^{6}$, SPIM $^{18}$, optoacoustic macroscopy using linear arrays ${ }^{17}$ and X-ray CT. However, MORSOM has unique features that lead to true mesoscopic optoacoustic imaging performance that has not previously been reported. For the first time, MORSOM offers ultra-broadband multi orientation mesoscopy that is appropriate for imaging model organisms. Increased imaging performance is achieved using a particular single-element ultra-wide band high-frequency detector design that enables multi orientation scanning over a $160-\mathrm{MHz}$ band for the $100-\mathrm{MHz}$ central frequency detector and a $\sim 92-\mathrm{MHz}$ band for the $50-\mathrm{MHz}$ central frequency detector. Ultrawide band detection is required to resolve the slow varying and fast varying spatial contributions, resulting in a resolution and overall image quality that is not possible using narrower bands. Combined with a complete $360^{\circ}$ angular coverage, MORSOM yielded highfidelity images of opaque biological tissues that were not accessible by optical and optoacoustic microscopy methods.

We chose single-element detectors because detector arrays might only achieve up to $35-\mathrm{MHz}$ bandwidths and have less preferential focusing characteristics. Therefore, MORSOM capitalizes on the collection of a uniquely rich and spatially precise data set, which we combined with a novel image reconstruction implementation to produce a higher SNR and higher and more isotropic in-plane resolution compared with RSOM.

The MORSOM-50 Zebrafish images exhibited higher contrast, richer anatomical detail and an improved SNR of $\sim 15 \mathrm{~dB}$ over RSOM, as shown in Figure $3 \mathrm{e}$ and $3 \mathrm{f}$,. MORSOM-100 images exhibited a higher resolution ( $\sim 9$ vs $17 \mu \mathrm{m}$ ) but a reduced SNR. Overall, MORSOM visualized diameters of up to $2 \mathrm{~mm}$, which are much deeper than what is allowed by SPIM or multiview optical resolution microscopy ${ }^{6}$. The latter operates with optical diffraction resolution using focused light beams that is a much higher resolution than optoacoustic mesoscopy using linear arrays ${ }^{15,16}$. The combination

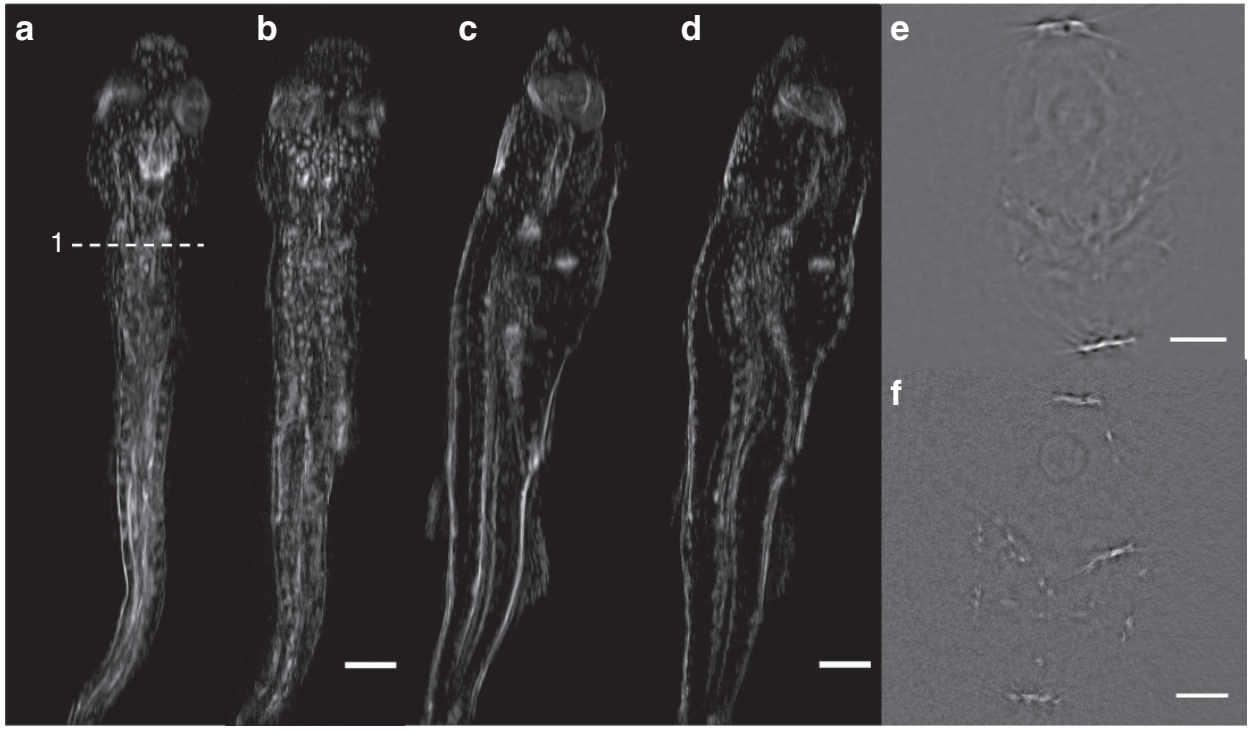

Figure 4 Comparison of MORSOM at $50 \mathrm{MHz}$ and at $100 \mathrm{MHz}$. (a) Side view MIP from MORSOM at $50 \mathrm{MHz}$. (b) Side view MIP from the $100 \mathrm{MHz}$ data. (c) Top view MIP from the $50 \mathrm{MHz}$ data. (d) Top view MIP from the $100 \mathrm{MHz}$ data. (e) Cross section through the Zebrafish at $50 \mathrm{MHz}$ compared with (f) $100 \mathrm{MHz}$. (Scale bars $=\mathbf{a}-\mathbf{d} 500 \mu \mathrm{m} ; \mathrm{e}, \mathrm{f} 250 \mu \mathrm{m}$ ). 
of advanced ultrasound detection techniques, including annular detector arrays ${ }^{27}$, could possibly improve the SNR and the focusing ability of the detector used.

A current limitation of MORSOM in comparison to RSOM is the scan duration, as several RSOM scans should be performed. A MORSOM scan at a $50 \mathrm{MHz}$ central frequency may take up to $20 \mathrm{~min}$ (up to $80 \mathrm{~min}$ for $100 \mathrm{MHz}$ ). This time scale does not limit the study of slow developmental processes occurring in juvenile and adult Zebrafish on a time scale of hours to days, e.g., organ development, growth of the vascular network, or changes in the neural network. Nevertheless, shorter time scales would be important for capturing faster phenomena and increasing throughput. The MORSOM scan times could be reduced by an optimized combination of $N_{\phi}$ and $N_{x}$ to retain the high-imaging speed of RSOM while adding the information from multiple orientations.

Further studies will focus on enabling multicolor imaging ${ }^{7}$, faster scanning and reconstruction times, and the combination of MORSOM with SPIM $^{28}$ or with other optical microscopic imaging modalities ${ }^{19}$ which will give complimentary contrast and complimentary imaging performance at different scales.

\section{CONFLICT OF INTEREST}

$\mathrm{VN}$ has a financial interest in iThera Medical GmbH, which did not support this work. KW, TSM and TM work for the ZEISS group, which is a manufacturer of optical microscopes.

\section{ACKNOWLEDGEMENTS}

We thank Laura Pola Morell for her help with the Zebrafish. We also thank Andrei Chekkoury for his valuable comments. We thank Dominik Soliman for his help with measuring the impulse response of the detectors. The work was sponsored by the Federal Ministry of Education and Research, Photonic Science Germany, Tech2See-13N12624.

1 Barbosa J, Sanchez-Gonzalez R, Di Giaimo R, Baumgart EV, Theis FJ et al. Live imaging of adult neural stem cell behavior in the intact and injured zebrafish brain. Science 2015; 348: 789-793.

2 Schönbauer C, Distler J, Jährling N, Radolf M, Dodt HU et al. Spalt mediates an evolutionarily conserved switch to fibrillar muscle fate in insects. Nature 2011; 479: 406-409.

3 Huisken J, Swoger J, Del Bene F, Wittbrodt J, Stelzer EHK. Optical sectioning deep inside live embryos by selective plane illumination microscopy. Science 2004; 305: 1007-1009.

4 Ntziachristos V. Going deeper than microscopy: the optical imaging frontier in biology. Nat Methods 2010; 7: 603-614.

5 Mahou P, Zimmerley M, Loulier K, Matho KS, Labroille G et al. Multicolor two-photon tissue imaging by wavelength mixing. Nat Methods 2012; 9: 815-818.

6 Zhu L, Li L, Gao L, Wang LV. Multiview optical resolution photoacoustic microscopy. Optica 2014; 1: 217-222.

7 Razansky D, Distel M, Vinegoni C, Ma R, Perrimon N et al. Multispectral opto-acoustic tomography of deep-seated fluorescent proteins in vivo. Nat Photonics 2009; 3 : 412-417.
8 Omar M, Gateau J, Ntziachristos V. Raster-scan optoacoustic mesoscopy in the 25-125 MHz range. Opt Lett 2013; 38: 2472-2474.

9 Omar M, Soliman D, Gateau J, Ntziachristos V. Ultrawideband reflection-mode optoacoustic mesoscopy. Opt Lett 2014; 39: 3911-3914.

10 Omar M, Schwarz M, Soliman D, Symvoulidis P, Ntziachristos V. Pushing the optical imaging limits of cancer with multi-frequency-band raster-scan optoacoustic mesoscopy (RSOM). Neoplasia 2015; 17: 208-214.

11 Tzoumas S, Zaremba A, Klemm U, Nunes A, Schaefer $\mathrm{K}$ et al. Immune cell imaging using multi-spectral optoacoustic tomography. Opt Lett 2014; 39: 3523-3526.

12 Wang LV, Hu S. Photoacoustic tomography: in vivo imaging from organelles to organs. Science 2012; 335: 1458-1462.

13 Beard P. Biomedical photoacoustic imaging. Interface Focus 2011; 1: 602-631.

14 Gamelin J, Aguirre A, Maurudis A, Huang F, Castillo D et al. Curved array photoacoustic tomographic system for small animal imaging. J Biomed Opt 2008; 13: 024007-024010.

15 Gateau J, Chekkoury A, Ntziachristos V. High-resolution optoacoustic mesoscopy with a $24 \mathrm{MHz}$ multidetector translate-rotate scanner. J Biomed Opt 2013; 18: 106005.

16 Li G, Li L, Zhu L, Xia J, Wang LV. Multiview Hilbert transformation for full-view photoacoustic computed tomography using a linear array. J Biomed Opt 2015; 20: 066010.

17 Chekkoury A, Gateau J, Driessen W, Symvoulidis P, Bézière N et al. Optical mesoscopy without the scatter: broadband multispectral optoacoustic mesoscopy. Biomed Opt Express 2015; 6: 3134-3148.

18 Huisken J, Stainier DY. Even fluorescence excitation by multidirectional selective plane illumination microscopy (mSPIM). Opt lett 2007; 32: 2608-2610.

19 Tserevelakis GJ, Soliman D, Omar M, Ntziachristos V. Hybrid multi-photon and optoacoustic microscope. Opt Lett 2014; 39: 1819-1822.

20 Soliman D, Tserevelakis GJ, Omar M, Ntziachristos V. Combining microscopy with mesoscopy using optical and optoacoustic label-free modes. Sci Rep 2015; 5: 12902.

21 Flood PM, Kelly R, Gutiérrez-Heredia L, Reynaud EG. ZEISS Lightsheet Z.1 Sample Preparation. Jena, Germany: Carl Zeiss Microscopy GmbH; 2013.

22 Yaroslavsky LP, Caulfield HJ. Deconvolution of multiple images of the same object. Appl Opt 1994; 33: 2157-2162.

23 Gustafsson MG, Shao L, Carlton PM, Wang CR, Golubovskaya IN et al. Threedimensional resolution doubling in wide-field fluorescence microscopy by structured illumination. Biophys J 2008; 94: 4957-4970.

24 Zhang C. Submicron-resolution photoacoustic microscopy of endogenous lightabsorbing biomolecules. Ph.D. thesis, Washington University, St. Louis, 2014.

25 Cobbold RSC Foundations of Biomedical Ultrasound. Oxford: Oxford University Press; 2007.

26 Kelsh RN, Brand M, Jiang YJ, Heisenberg CP, Lin S et al. Zebrafish pigmentation mutations and the processes of neural crest development. Development 1996; 123 : 369-389.

27 Chitnis PV, Aristizábal O, Filoux E, Sampathkumar A, Mamou J et al. Coherenceweighted synthetic focusing applied to photoacoustic imaging using a high-frequency annular-array transducer. Ultrason Imaging 2016; 38: 32-43.

28 Lin HCA, Chekkoury A, Omar M, Schmitt-Manderbach T, Koberstein-Schwarz B et al. Selective plane illumination optical and optoacoustic microscopy postembryonic imaging. Laser Photon Rev 2015; 9: L29-L34.

cc) $(-)$ This work is licensed under a Creative Commons Attributioncc) $\mathrm{BY}$ NC ND NonCommercial-NoDerivs 4.0 International License. The images or other third party material in this article are included in the article's Creative Commons license, unless indicated otherwise in the credit line; if the material is not included under the Creative Commons license, users will need to obtain permission from the license holder to reproduce the material. To view a copy of this license, visit http://creativecommons.org/ licenses/by-nc-nd/4.0/

(C) The Author(s) 2017

Supplementary Information for this article can be found on the Light: Science \& Applications' website (http://www.nature.com/lsa). 